\title{
Gambogenic acid inhibits the proliferation of small-cell lung cancer cells by arresting the cell cycle and inducing apoptosis
}

\author{
TINGTING HUANG ${ }^{1-3^{*}}$, HONGMING ZHANG ${ }^{4 *}$ XIYONG WANG ${ }^{2,3}, \mathrm{LU} \mathrm{XU}^{2,3}$, \\ JINFANG JIA ${ }^{2,3}$ and XIAOLI ZHU ${ }^{2,3}$ \\ ${ }^{1}$ Department of Respiratory Medicine, The First People's Hospital of Lianyungang, Lianyungang, Jiangsu 222000; \\ ${ }^{2}$ Department of Respiratory Medicine, Zhongda Hospital, Southeast University; ${ }^{3}$ Medical School of Southeast University, \\ Nanjing, Jiangsu 210009; ${ }^{4}$ Department of Respiratory Medicine, Yancheng Third People's Hospital, \\ The Affiliated Yancheng Hospital of Southeast University Medical College, \\ Yancheng, Jiangsu 224001, P.R. China
}

Received November 8, 2017; Accepted December 13, 2018

DOI: $10.3892 /$ or.2018.6950

\begin{abstract}
Gambogenic acid (GNA), which is an important active compound present in gamboge, exerts anticancer activity in various types of tumor cells. However, the effect of GNA on small-cell lung cancer (SCLC) cell lines and the underlying mechanism involved still remain unclear. In the present study, GNA inhibited the proliferation and cell cycle progression of SCLC cells. GNA also promoted the apoptosis of SCLC cells in a dose-dependent manner, which is associated with modulating the levels of proteins involved in apoptosis pathways in NCI-H446 and NCI-H1688 cells. The results demonstrated that GNA increased the level of cleaved caspase-3, -8 and -9 , and Bax but decreased the expression of anti-apoptotic protein, Bcl-2. Furthermore, similar results were obtained in a mouse tumor xenograft model. Additionally, GNA exhibit low toxicity in tissues when administered to mice in the SCLC xenograft models. Collectively, our findings demonstrated that GNA significantly inhibited the proliferation of SCLC cells and promoted cell apoptosis via cell cycle arrest and induction of apoptosis.
\end{abstract}

\section{Introduction}

Lung cancer is one of the main causes of cancer-associated mortality worldwide. The two major histopathological groups of lung cancer are non-small cell lung cancer (NSCLC), which accounts for $80-85 \%$ of cases, and small-cell lung cancer

Correspondence to: Mrs. Xiaoli Zhu, Department of Respiratory Medicine, Zhongda Hospital, Southeast University, 87 Dingjiaqiao, Gulou, Nanjing, Jiangsu 210009, P.R. China

E-mail: zhuxiaoli62@163.com

${ }^{*}$ Contributed equally

Key words: gambogenic acid, proliferation, cell cycle, apoptosis, small-cell lung cancer cells
(SCLC), which accounts for $15-20 \%$ of cases $(1,2)$. SCLC is an aggressive lung cancer subtype characterized by rapid expansion and metastasis of cells with neuroendocrine features. Presently, platinum-based chemotherapy is the first-line treatment for patients with SCLC; however, patients have a low median survival time $(9-12$ months) $(3,4)$, and experience high toxicity and intolerance. In addition, recurrence occurs rapidly in the majority of patients with SCLC, and patients with recurrence have a median survival of only 4-5 months when treated with further systemic therapy (5). No targeted drugs are currently used as SCLC treatments. Therefore, novel therapeutic strategies for SCLC are urgently needed.

Gambogenic acid (GNA) is an active compound isolated from gamboge, which is a resin exuded from the Garcinia hanburyi tree $(6,7)$. GNA has been reported to have more potent anticancer effects and less systemic toxicity than gambogic acid (GA), another active compound present in gamboge (8-10). Induction of apoptosis has been characterized as the main molecular and biochemical effect of GNA in various cancer cell lines and animal models of carcinogenesis (11-15). GNA inhibits the proliferation of A549 cells by inducing cell apoptosis and cell cycle arrest (13). GNA can also cause glycogen synthase kinase $3 \beta$-dependent G1 arrest in lung cancer cells (16). Although several studies have reported the anticancer activity of GNA in NSCLC (11-17), whether GNA can exert antitumor effects in SCLC remains unknown.

In the present study, we aimed to investigate the effects of GNA on SCLC in vitro and in vivo. Notably, we found that GNA significantly suppressed the proliferation of SCLC cells and cell cycle progression. GNA also promoted cell apoptosis and modulated the expression of apoptosis-related proteins. The findings indicated that GNA may be a useful therapeutic reagent for SCLC treatment.

\section{Materials and methods}

Reagents. RPMI-1640, fetal bovine serum (FBS) and penicillin/streptomycin were purchased from Thermo Fisher Scientific, Inc. (Waltham, MA, USA). Dimethyl sulfoxide (DMSO) was obtained from Sigma-Aldrich (Merck KGaA, 
Darmstadt, Germany), The Annexin V-FITC Apoptosis Detection kit and the TUNEL Apoptosis Assay kit were purchased from BD Biosciences (San Jose, CA, USA). Cell Counting Kit-8 (CCK-8) was purchased from Dojindo Molecular Technologies, Inc. (Kumamoto, Japan). All antibodies were purchased from Cell Signaling Technology, Inc. (Danvers, MA, USA).

Cell culture and drug treatment. The human SCLC cell lines, NCI-H446 and NCI-H1688, were purchased from the Cell Bank of the Chinese Academy of Sciences (Shanghai, China). The cells were cultured in RPMI-1640 medium supplemented with $10 \% \mathrm{FBS}, 1 \times 10^{5} \mu \mathrm{g} / \mathrm{ml}$ penicillin and $1 \times 10^{5} \mu \mathrm{g} / \mathrm{ml}$ streptomycin at $37^{\circ} \mathrm{C}$ in a humidified atmosphere containing $5 \%$ carbon dioxide. GNA (Fig. 1; purity $>98 \%$; Ronghe Medical Technology, Shanghai, China) was dissolved in DMSO to $100 \mathrm{mM}$ as stock solution, and the final concentration of DMSO was $<0.1$ percent and stored at $-20^{\circ} \mathrm{C}$.

CCK- 8 assay. NCI-H446 and NCI-H1688 $\left(4 \times 10^{3}\right)$ cells were seeded into 96-well plates, respectively. Then various concentrations of GNA (NCI-H446: 0, 0.6, 1.0, 1.4, 1.8, 2.2 and $2.4 \mu \mathrm{M}$; NCI-H1688: 0, 1.2, 1.6, 2.0, 2.4, 2.8 and $3.2 \mu \mathrm{M})$ was added for 24, 48 and $72 \mathrm{~h}$. Cell Counting Kit-8 (CCK-8) reagent was added to each tested well of the plate after incubation with several concentrations of GNA for 24, 48 and $72 \mathrm{~h}$, and then incubated for $4 \mathrm{~h}$ at $37^{\circ} \mathrm{C}$. The optical density at $450 \mathrm{~nm}$ was detected using a microplate reader (Bio-Rad Laboratories, Inc., Hercules, CA, USA). A total of three independent experiments were performed.

Cell cycle analysis. SCLC cells were treated with various concentrations of GNA (NCI-H446: $0,0.6$ and $1.2 \mu \mathrm{M}$; NCI-H1688: $0,1.5$ and $2.2 \mu \mathrm{M}$ ) for $24 \mathrm{~h}$. After $24 \mathrm{~h}$, the cells were harvested and fixed in cold $70 \%$ ethanol. The cells were then incubated with propidium iodide (PI; Cell Cycle kit; BD Biosciences) and then analyzed by flow cytometry (FACSCalibur; BD Biosciences). Three independent experiments were performed.

Cell apoptosis assay. SCLC cells were treated with various concentrations of GNA (NCI-H446: $0,1.4$ and $2.0 \mu \mathrm{M}$; NCI-H1688: 0, 2.4 and $3.0 \mu \mathrm{M}$ ) for $24 \mathrm{~h}$. After $24 \mathrm{~h}$, the cells were trypsinized and washed with phosphate-buffered saline (PBS). Then, the cells were incubated with binding buffer containing Annexin V-FITC and PI according to the manufacturer's protocol (Annexin V-FITC Apoptosis Detection kit; BD Biosciences) and then analyzed by flow cytometry (FACSCalibur; BD Biosciences). Three independent experiments were performed.

Xenograft nude mouse model. Eighteen BALB/c nude male mice at 4-6 weeks-old, with body weight of 18-22 g, were purchased from the Vital River Laboratory Animal Technology Co., Ltd. (Beijing, China) and randomly divided into three groups. Each group consisted of six mice. The mice were raised in specific pathogen-free environment, with 12-h light/dark cycles and free access to standard rodent food and water. NCI-H446 cells $\left(1 \times 10^{6}\right)$ were suspended in $100 \mu \mathrm{l}$ PBS and injected subcutaneously in the left armpit of mice.

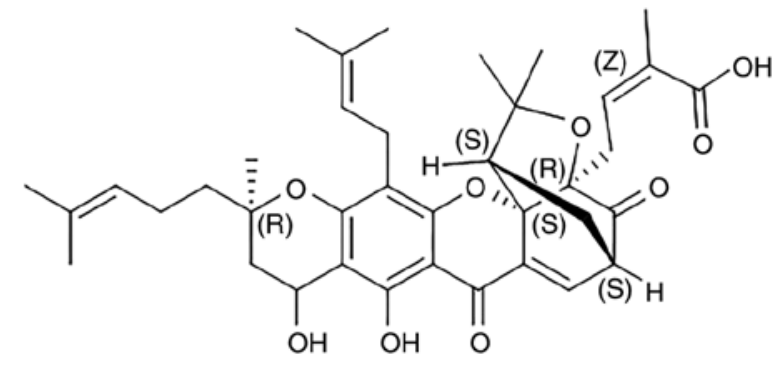

Figure 1. Chemical structure of gambogenic acid.

Once the tumors were established $\left(\sim 1 \mathrm{~cm}^{3}\right)$, the mice were injected intravenously with different GNA concentrations ( 4 and $12 \mathrm{mg} / \mathrm{kg}$ ) or normal saline at 1 p.m. every 2 days. The total injection time for all mice was $30 \mathrm{~min}$. The weight of the mice and tumor sizes were recorded 2 days before and after GNA treatment. The tumor volume was calculated using the formula: length $\mathrm{x}\left(\right.$ width $\left.^{2}\right) / 2$. Careful daily rationing, weighing out how much food was left and assessing their appetite was performed. When the mice developed loss of appetite, all of them were intravenously anesthetized by $1 \%$ pentobarbitone sodium $(30 \mathrm{mg} / \mathrm{kg})$ and then sacrificed by decapitation. The maximum tumor diameter was $15 \mathrm{~mm}$ (tumor weight percentage: $9 \%$ ). Nude mice were sacrificed on the 28th day after the administration, and the tumor body was peeled and weighed. Concurrently, the heart, liver, spleen, lung and kidney organs were dissected. After three washes with saline, a portion of the tumors were immersed in the liquid nitrogen and then transferred to a $-80^{\circ} \mathrm{C}$ refrigerator for TUNEL detection, and the remaining portion of the tumor and the organs were placed in $4 \%$ paraformaldehyde solution for subsequent western blotting and hematoxylin and eosin (H\&E) detection. In the experiment, there were no multiple tumors in nude mice. All of the protocols were approved by the Animal Ethics Committee of the Medical School, Southeast University (Nanjing, China).

Western blotting. The expression of apoptosis-related proteins following treatment with GNA were analyzed by western blotting. In vitro, NCI-H446 cells were respectively treated with $0,1.1$ and $1.3 \mu \mathrm{M}$ GNA, and the concentration was $0,2.0$ and $2.3 \mu \mathrm{M}$ for NCI-H1688 cells. The dose of the drug was decided at the beginning of the in vivo xenograft nude mouse model. The cells and tumor tissue protein were lysed on ice in RIPA buffer (Nanjing KeyGen Biotech Co., Ltd., Nanjing, China) containing a protease inhibitor cocktail (Merck KGaA) and were quantified using a BCA Assay kit (Thermo Fisher Scientific, Inc.). The proteins lysates were then separated by 8-12\% SDS-PAGE and transferred to polyvinylidene difluoride membranes (EMD Millipore, Billerica, MA, USA). The membranes were blocked in $5 \%$ non-fat milk and were incubated overnight at $4^{\circ} \mathrm{C}$ with diluted $(1: 1,000)$ specific primary antibodies against caspase-3 (cat. no. 9664), -8 (cat. no. 9496) and caspase-9 (cat. no. 52873), Bax (cat. no. 5023), Bcl-2 (cat. no. 15071), p53 (cat. no. 2527), poly[ADP-ribose] polymerase 4 (PARP) (cat. no. 5625), $\beta$-actin (cat. no. 4970) (Cell Signaling Technology, Inc.). Subsequently, the membranes were washed with TBST buffer and were incubated with the appropriate secondary antibodies (dilution 1:5,000; 

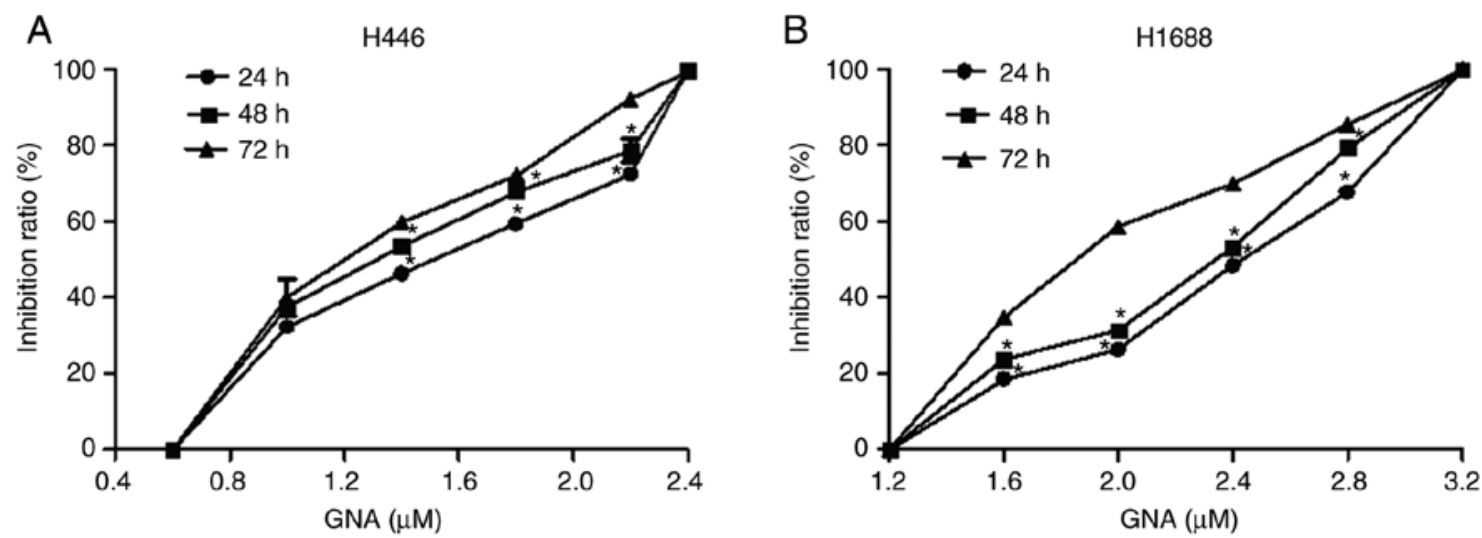

Figure 2. Proliferation of small-cell lung cancer cell lines is inhibited by GNA. (A) NCI-H446 and (B) NCI-H1688 cells were treated with various concentrations of GNA for 24,48 and $72 \mathrm{~h}$. The inhibition rate was detected by CCK- 8 assay. Data are presented as the mean \pm standard deviation $\left(\mathrm{n}=3\right.$ ); ${ }^{*} \mathrm{P}<0.05$. GNA, gambogenic acid; CCK-8, Cell Counting Kit-8.

anti-rabbit IgG: cat. no. 14708; anti-mouse IgG: cat. no. 58802; Santa Cruz Biotechnology, Inc., Dallas, TX, USA) for $1 \mathrm{~h}$ at room temperature. The blots were detected using ECL reagents (Thermo Fisher Scientific, Inc.). $\beta$-actin and GAPDH were used as loading controls. Three independent experiments were performed and the ImageJ (version $1.44 \mathrm{p}$ analysis system; NIH; National Institute of Mental Health, Bethesda, MD, USA) was used to measure the intensity of the bands.

Transferase dUTP nick end-labeling (TUNEL) analysis. A TUNEL assay was performed by using In Situ Cell Death Detection kit (BD Biosciences) following the manufacturer's protocol. Fluorescence emitted from tissue sections was analyzed, and the images were captured using a fluorescence microscope (Nikon Corp., Tokyo, Japan).

Histological analysis. The lung, liver, kidney, spleen and heart tissues from the SCLC xenograft model mice were fixed in $10 \%$ paraformaldehyde and stained with H\&E. Histopathological changes were observed by light microscopy.

Statistical analysis. All the data are presented as the mean \pm standard deviation of three independent experiments. One-way analysis of variance (ANOVA) followed by Dunnett's test were used to analyze the data. Differences were considered statistically significant at $\mathrm{P}<0.05$.

\section{Results}

GNA inhibits the proliferation of SCLC cell lines. CCK-8 assay results demonstrated that GNA significantly suppressed the proliferation of NCI-H446 cells at $0.6-2.4 \mu \mathrm{M}$ in a time- and dose-dependent manner. The $\mathrm{IC}_{50}$ in NCI-H446 cells was $1.4 \mu \mathrm{M}$ (Fig. 2A). Additionally, the suppressive effect of GNA on the proliferation of NCI-H1688 cells was time- and dose-dependent at 1.2-3.2 $\mu \mathrm{M}$ with an $\mathrm{IC}_{50}$ value of $2.4 \mu \mathrm{M}$ (Fig. 2B).

Effect of GNA on the cell cycle in SCLC cells. To determine whether the inhibition of SCLC cell proliferation by GNA was mediated by cell cycle arrest, the cell cycle phases were examined in NCI-H446 and NCI-H1688 cell lines that were treated with different concentrations of GNA. For NCI-H446, the data revealed that low doses of GNA arrested cell cycle progression in the G0/G1 phase, while higher concentrations blocked the cycle in the $\mathrm{S}$ phase (Fig. 3A). However, the same effect was not observed in NCI-H1688 cells, which was blocked at the $\mathrm{S}$ phase in a dose-dependent manner (Fig. 3B). Therefore, higher concentrations of GNA inhibited cell cycle progression in SCLC cell lines by inducing arrest at the $S$ phase. According to ANOVA and Dunnett's test, significant differences were observed between the experimental groups and the control group.

GNA promotes apoptosis in SCLC cell lines. GNA has been previously revealed to inhibit proliferation and induce apoptosis of A549 cells (11); therefore, experiments were performed to verify whether GNA had similar effects on NCI-H446 and NCI-H1688 cells. The results demonstrated that GNA increased the rate of cell apoptosis in a dose-dependent manner (Fig. 4A and B), indicating that GNA induces apoptosis in SCLC cell lines. According to ANOVA and Dunnett's test, significant differences were observed between the experimental groups and the control group.

GNA activates apoptotic pathways in SCLC cells. It was subsequently examined whether GNA-induced apoptosis of SCLC cells was mediated by activating apoptotic pathways. After treatment with different concentrations of GNA for $24 \mathrm{~h}$, the expression of apoptosis-related proteins was detected by western blotting. The expression of cleaved caspase- $3,-8$ and -9 , Bax, cleaved PARP and p53 proteins were significantly increased in the GNA treatment groups compared with the control group. However, Bcl-2 expression was decreased in GNA treatment groups compared with the control group (Fig. 5). Therefore, GNA may induce cell apoptosis by increasing the level of pro-apoptosis proteins and inhibiting the expression of anti-apoptosis proteins in SCLC cell lines. According to ANOVA and Dunnett's test, significant differences were observed between the experimental groups and the control group.

GNA suppresses tumor growth in vivo. Subsequently, experiments were designed to further investigate whether GNA 

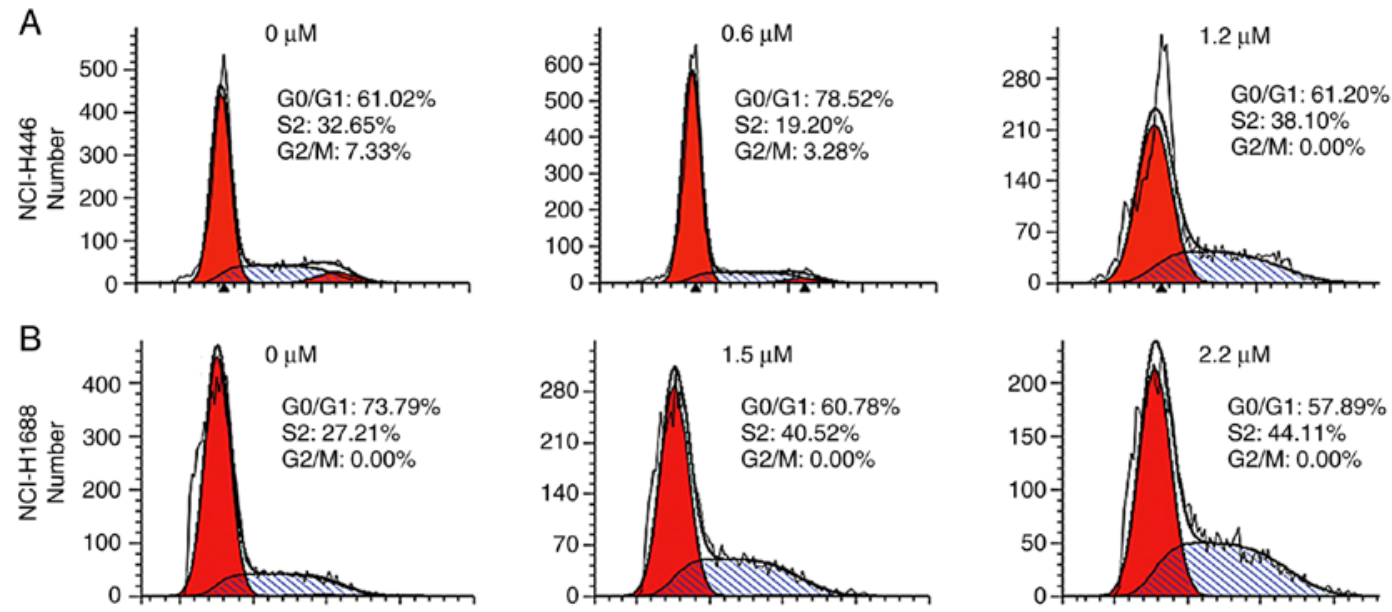

Figure 3. GNA arrests cell cycle progression in different phases. Cell cycle distribution was analyzed by flow cytometry after $24 \mathrm{~h}$ of GNA treatment. (A) NCI-H446 cells were arrested at different stages by various drug concentrations. (B) NCI-H1688 cell cycle was arrested by GNA during the S stage. Data are presented as the mean \pm standard deviation, $(\mathrm{n}=3)$; GNA, gambogenic acid.

A

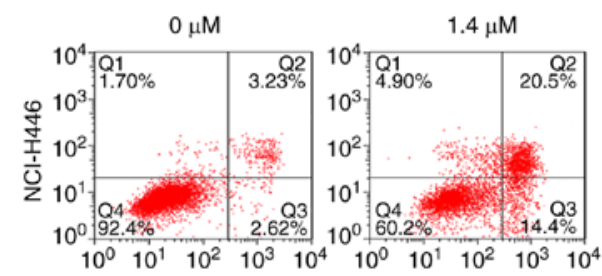

B

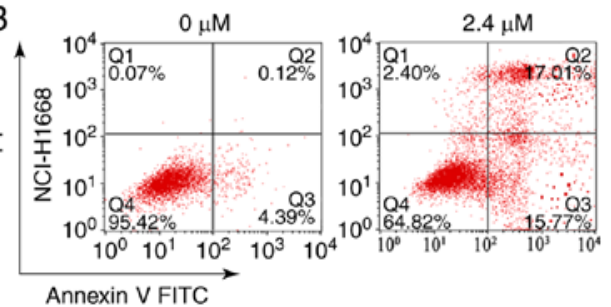

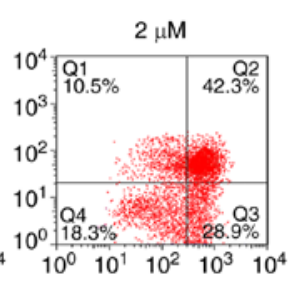
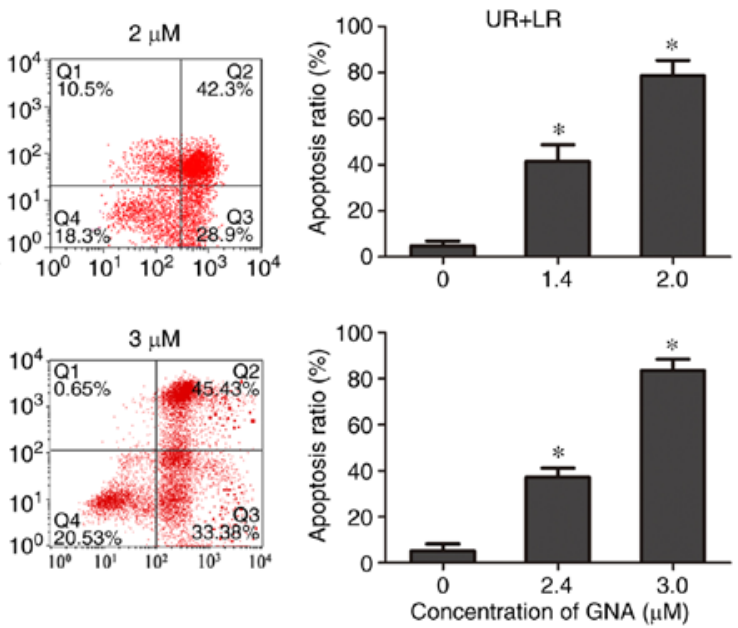

Figure 4. GNA induces apoptosis of small-cell lung cancer cell lines. Cell apoptosis was determined using Annexin V/PI staining assay and flow cytometry. (A) NCI-H446 and (B) NCI-H1688 cells were treated with various GNA concentrations for $24 \mathrm{~h}$ and a significant dose-dependent increase in the proportion of apoptotic cells was observed. Data are presented as the mean \pm standard deviation, $(n=3)$; ${ }^{*} \mathrm{P}<0.05$. GNA, gambogenic acid; UR, upper right; LR, lower right.
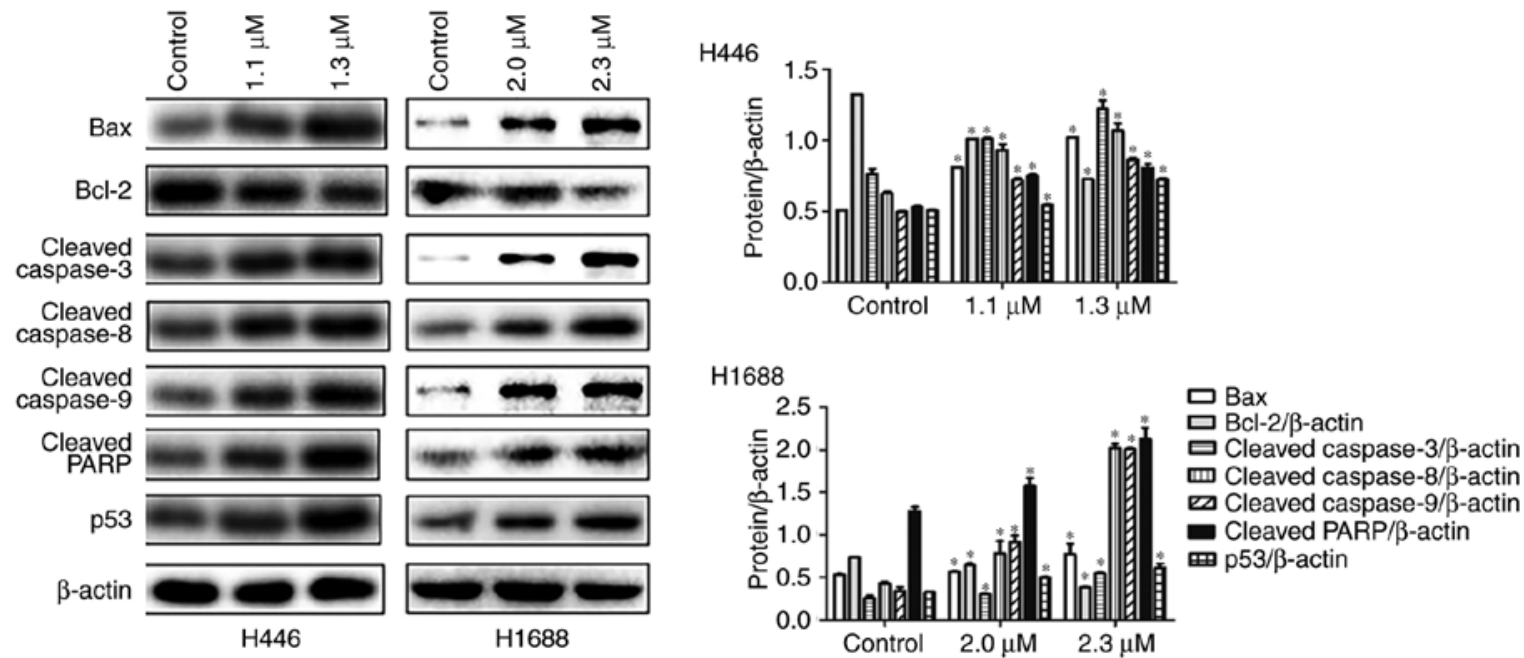

Figure 5. GNA promotes the expression of pro-apoptotic proteins. The expression of apoptosis-related proteins was analyzed by western blotting. NCI-H446 cells were treated with different GNA concentrations $(0,1.1$ and $1.3 \mu \mathrm{M})$ for $24 \mathrm{~h}$; NCI-H1688 cells were treated with different GNA concentrations $(0,2.0$ and $2.3 \mu \mathrm{M})$ for $24 \mathrm{~h}$, and the expression levels of cleaved caspase-3, -8 and -9, Bax, Bcl-2 and cleaved PARP were detected by western blotting. $\beta$-actin was used as an internal control. Data are presented as the mean \pm standard deviation $(n=3) ;{ }^{*} \mathrm{P}<0.05$. GNA, gambogenic acid; PARP, poly(ADP-ribose) polymerase 4 . 


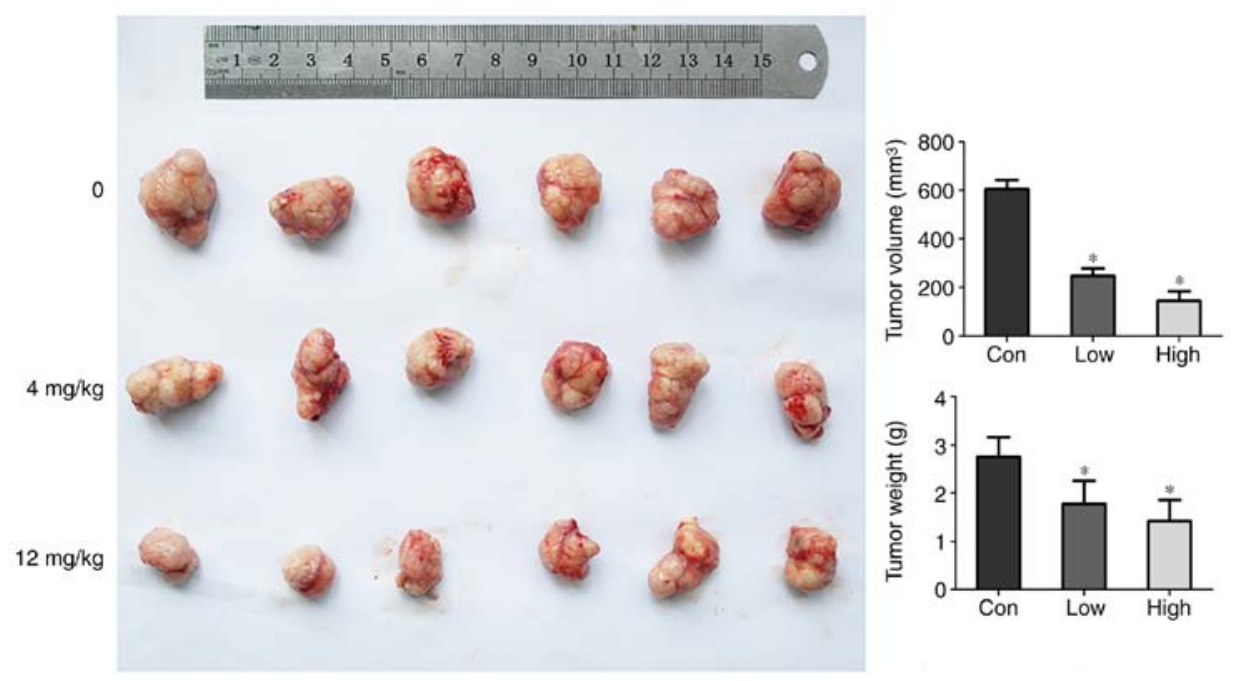

Figure 6. GNA inhibits tumor growth in an SCLC mouse xenograft model. The nude mice that were transplanted with SCLC xenografts were randomly divided into three groups: Con, control group; Low, Low treatment group ( $4 \mathrm{mg} / \mathrm{kg}$ every 2 days); and High, high treatment group (12 mg/kg every 2 days). Treatment was administered by intravenous injection of GNA or vehicle for 14 days. The tumors in each group were dissected and imaged. The measurements of tumor volumes are presented. The body weight and tumor volumes of each group are presented as the mean \pm standard deviation $(\mathrm{n}=6)$, ${ }^{*} \mathrm{P}<0.05$. GNA, gambogenic acid; SCLC, small cell lung cancer.

A

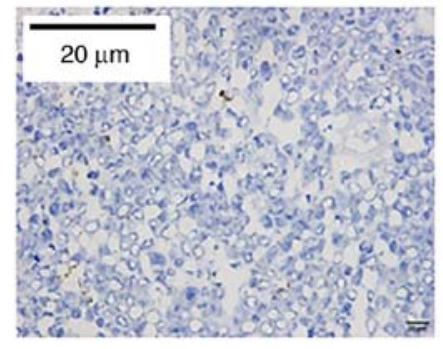

B
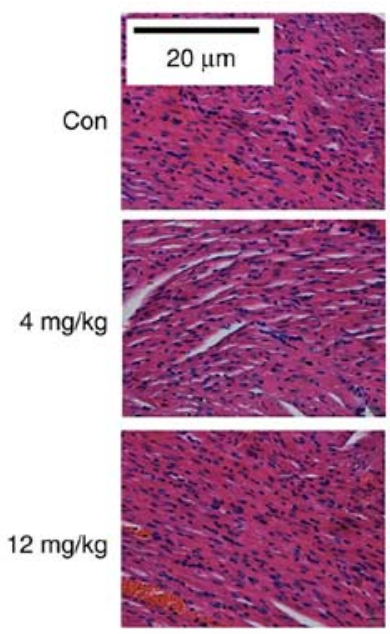

Heart

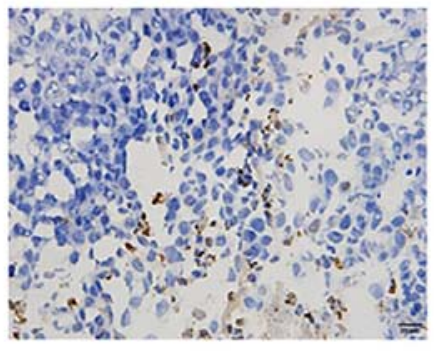

$4 \mathrm{mg} / \mathrm{kg}$

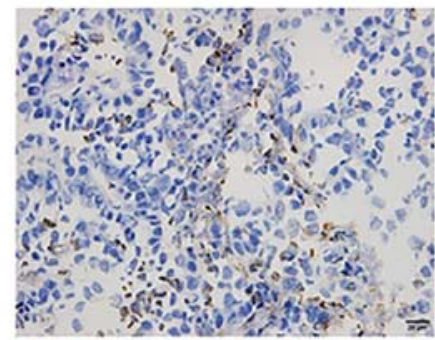

$12 \mathrm{mg} / \mathrm{kg}$
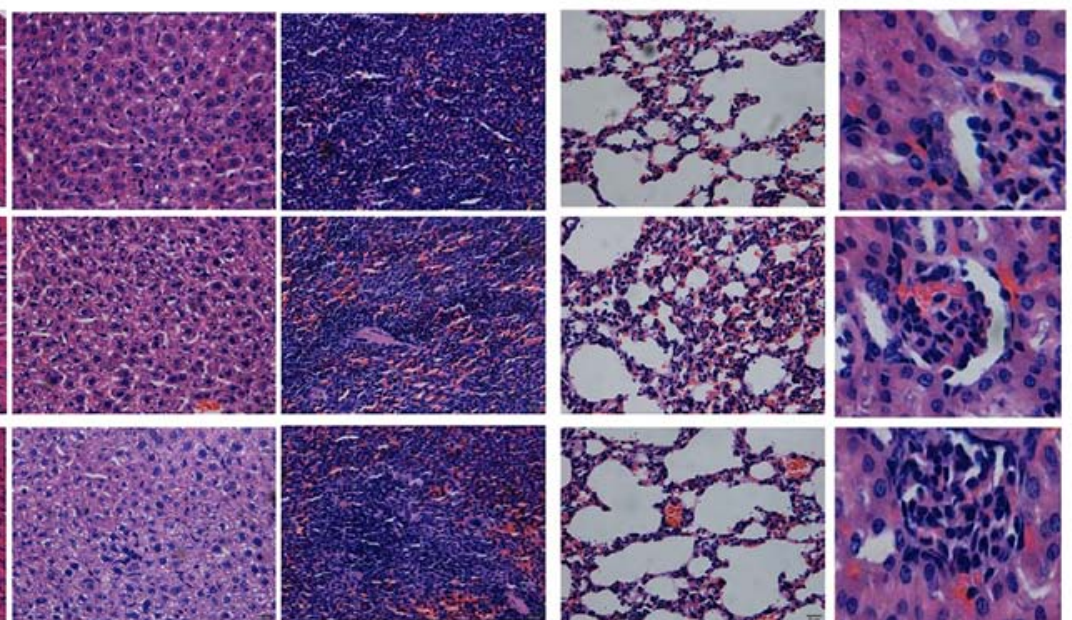

Liver

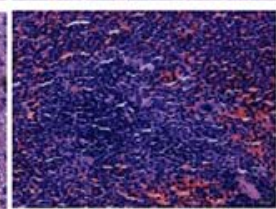

Spleen

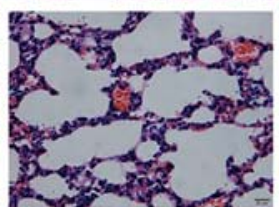

Lung

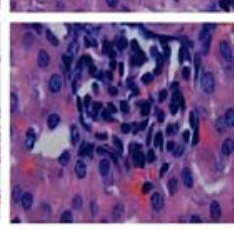

Kidney

Figure 7. GNA induces apoptosis in mouse xenograft models of SCLC. (A) TUNEL staining was performed on histological sections of tumor tissue to detect apoptotic cells. Positive TUNEL staining was observed as light yellow to brownish yellow granules in the cytoplasm. (B) H\&E staining was performed to assess toxicity in the lung, liver, kidney, spleen and heart of xenograft mice that were treated with GNA. Data are presented as the mean \pm standard deviation $(\mathrm{n}=6)$. GNA, gambogenic acid; SCLC, small-cell lung cancer; TUNEL, transferase dUTP nick end-labeling; H\&E, hematoxylin and eosin.

inhibited the growth of SCLC tumors in vivo. GNA-treated mice exhibited tumor tissue loss compared with the control group (Fig. 6). According to ANOVA and Dunnett's test, significant differences were observed between experimental groups and the control group. TUNEL staining further validated that
GNA induced apoptosis in a dose-dependent manner (Fig. 7A), which was consistent with the studies performed in vitro. Furthermore, in the in vivo tumor study, the expression of apoptosis-related proteins exhibited the same trends as observed in vitro (Fig. 8). The expression of pro-apoptotic 


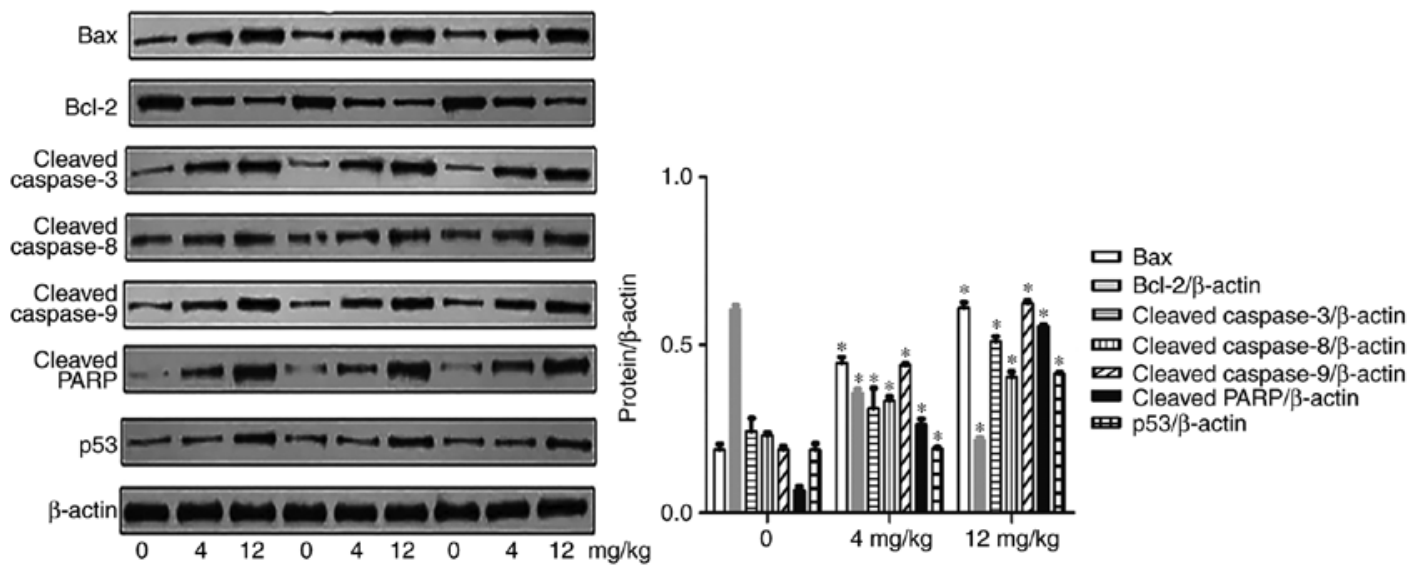

Figure 8. GNA modulates the levels of apoptosis-related proteins in mouse small-cell lung cancer xenograft tissues. (A) Protein level of cleaved caspase-3, -8 and -9 , Bax, Bcl-2, cleaved PARP and p53 were analyzed by western blotting. GAPDH was used as an internal control. (B) Data are presented as the mean \pm standard deviation $(\mathrm{n}=6) ;{ }^{*} \mathrm{P}<0.05$. GNA, gambogenic acid; PARP, poly[ADP-ribose] polymerase 4.

proteins was increased, while the anti-apoptotic proteins were decreased in the GNA treatment groups compared with the control group. These findings further demonstrated that GNA could induce cell apoptosis by activating apoptosis-related proteins in SCLC cell lines. According to ANOVA and Dunnett's test, significant differences were observed between the experimental groups and the control group.

GNA has a low toxicity in vivo. H\&E staining of tissues collected from the xenograft mice was conducted to detect toxicity in vivo. No apoptotic cell death was observed in the lung, liver, kidney, spleen or heart tissues from the SCLC xenograft mice (Fig. 7B).

\section{Discussion}

The clinical applications of traditional Chinese medicine in antitumor research has been extensively investigated $(19,20)$. Among them, GNA has been reported to exert numerous pharmacological activities, particularly broad-spectrum anticancer effects $(21,18)$. In the present study, our results illustrated that GNA inhibited cell proliferation, induced cell apoptosis and induced cell cycle arrest in SCLC cells. Furthermore, GNA suppressed tumor growth in a mouse SCLC xenograft model.

Previous studies have reported that many active compounds present in traditional Chinese medicines can arrest the cell cycle in tumor cells, including GNA (14), wogonin (18) and curcumin (22). GNA was previously reported to induce G1 arrest in non-small cell lung cancer cells $(12,13,16)$ and breast cancer cells (22). GNA also induced G2/M phase arrest in multiple myeloma cells (23). Notably, our results revealed that low doses of GNA arrested cell cycle progression in the G0/G1 phase, whereas a higher concentration caused S-phase arrest of NCI-H446 SCLC cells. This suggests that the underlying mechanisms of GNA-induced cell cycle are complex, and further studies are required to understand this phenomenon.

GNA was revealed to inhibit cell proliferation by inducing apoptosis in non-small cell lung cancer cells $(13,16)$. GNA was revealed to induce apoptosis and G0/G1 phase arrest of A549 cells in a dose- and time-dependent manner in vitro. Apoptosis is a programmed cell death mechanism and is the most common form of cell death $(24,25)$. In the present study, we observed that cleaved caspase- $3,-8,-9$, Bax, cleaved PARP and p53 protein levels were increased by GNA treatment, whereas Bcl-2 expression was decreased. Our findings demonstrated that GNA promoted the apoptosis of SCLC cells by upregulating pro-apoptotic mediators in vitro and in vivo. It is important to highlight that no toxicity was observed following the treatment of nude mice with GNA, which was confirmed by $H \& E$ staining of the mouse lung, liver, tumor, kidney, spleen and heart tissues (26).

The present study has a number of limitations. The exact mechanism by which GNA induces cell cycle arrest and apoptosis remains unknown $(16,18,27)$. Given the important effects of GNA on inhibiting the proliferation of SCLC cells, the effective concentrations and potential mechanism of GNA in SCLC cells remain to be investigated in future studies.

In conclusion, the findings, based on the experiments in vivo and in vitro, demonstrated that GNA could inhibit cell proliferation, induce cell apoptosis and cell cycle arrest in two SCLC cell lines. In addition, the growth of SCLC xenograft tumors was also suppressed by GNA. Furthermore, GNA modulated the levels of apoptosis-related proteins and promoted the apoptosis of SCLC cells in vitro and in vivo, and exhibited a relatively low toxicity in mice, which was clearly different from GA (26). In conclusion, GNA may be a potential candidate for treatment of SCLC.

\section{Acknowledgements}

The present study was supported by the Innovation Program for College Graduates of Jiangsu Province, People's Republic of China (no. SJCX17_0058).

\section{Funding}

No funding was received.

\section{Availability of data and materials}

The datasets used or analysed during the current study are available from the corresponding author on reasonable request. 


\section{Authors' contributions}

$\mathrm{TH}$ and $\mathrm{HZ}$ acquired the data and created a draft of the manuscript; XW, LX and JJ prepared the experimental materials and performed the in vitro assays; XZ, TH and HZ processed the interpreted data, performed the statistical analysis and analyzed the results; XZ, HZ and TH revised and approved the final version of the manuscript. All authors read and approved the manuscript and agree to be accountable for all aspects of the research in ensuring that the accuracy or integrity of any part of the work are appropriately investigated and resolved.

\section{Ethics approval and consent to participate}

All the animal experiments were conducted under protocols approved by the Animal Ethics Committee of the Medical School, Southeast University.

\section{Patient consent for publication}

Not applicable.

\section{Competing interests}

The authors declare that they have no competing interests.

\section{Authors' information}

Xiaoli Zhu, MD, Chair, Department of Pulmonary Medicine, Zhongda Hospital, Medical School, Southeast University, China. She has published $>10$ SCI-indexed papers in some renowned journals, such as FEBS Journal and presided over a number of provincial subjects. In addition, she has published several influential studies and has held a variety of academic posts.

\section{References}

1. van Meerbeeck JP, Fennell DA and De Ruysscher DK: Small-cell lung cancer. Lancet 378: 1741-1755, 2011.

2. Heist RS and Engelman JA: SnapShot: Non-small cell lung cancer. Cancer Cell 21: 448 e442, 2012.

3. Noda K, Nishiwaki Y, Kawahara M, Negoro S, Sugiura T, Yokoyama A, Fukuoka M, Mori K, Watanabe K, Tamura T, et al: Irinotecan plus cisplatin compared with etoposide plus cisplatin for extensive small-cell lung cancer. N Engl J Med 346: 85-91, 2002.

4. Lara PN Jr, Natale R, Crowley J, Lenz HJ, Redman MW, Carleton JE, Jett J, Langer CJ, Kuebler JP, Dakhil SR, et al: Phase III trial of irinotecan/cisplatin compared with etoposide/ cisplatin in extensive-stage small-cell lung cancer: Clinical and pharmacogenomic results from SWOG S0124. J Clin Oncol 27: 2530-2535, 2009.

5. Network NCC: NCCN Clinical Practice Guidelines in Oncology (NCCN Guidelines ${ }^{\circledR}$ ), 2018

6. Asano J, Chiba K, Tada M and Yoshii T: Cytotoxic xanthones from Garcinia hanburyi. Phytochemistry 41: 815-820, 1996

7. Song JZ, Yip YK, Han QB, Qiao CF and Xu HX: Rapid determination of polyprenylated xanthones in gamboge resin of Garcinia hanburyi by HPLC. J Sep Sci 30: 304-309, 2007.

8. Lin T, Fang Q, Peng D, Huang X, Zhu T, Luo Q, Zhou K and Chen W: PEGylated non-ionic surfactant vesicles as drug delivery systems for gambogenic acid. Drug Deliv 20: 277-284, 2013.
9. Chen HB, Zhou LZ, Mei L, Shi XJ, Wang XS, Li QL and Huang L: Gambogenic acid-induced time- and dose-dependent growth inhibition and apoptosis involving Akt pathway inactivation in U251 glioblastoma cells. J Nat Med 66: 62-69, 2012.

10. Chen F, Zhang XH, Hu XD, Zhang W, Lou ZC, Xie LH, Liu PD and Zhang HQ: Enhancement of radiotherapy by ceria nanoparticles modified with neogambogic acid in breast cancer cells. Int J Nanomedicine 10: 4957-4969, 2015.

11. Yang L, Wang M, Cheng H and Li Q: Gambogenic acid inhibits proliferation of A549 cells through apoptosis-inducing. Zhongguo Zhong Yao Za Zhi 36: 1217-1221, 2011 (In Chinese).

12. Cheng H, Su JJ, Peng JY, Wang M, Wang XC, Yan FG, Wang XS and Li QL: Gambogenic acid inhibits proliferation of A549 cells through apoptosis inducing through up-regulation of the p38 MAPK cascade. J Asian Nat Prod Res 13: 993-1002, 2011.

13. Li Q, Cheng H, Zhu G, Yang L, Zhou A, Wang X, Fang N, Xia L, $\mathrm{Su}$ J, Wang M, et al: Gambogenic acid inhibits proliferation of A549 cells through apoptosis-inducing and cell cycle arresting. Biol Pharm Bull 33: 415-420, 2010.

14. Yan F, Wang M, Li J, Cheng H, Su J, Wang X, Wu H, Xia L, Li X, Chang HC, et al: Gambogenic acid induced mitochondrial-dependent apoptosis and referred to phospho-Erk1/2 and phospho-p38 MAPK in human hepatoma HepG2 cells. Environ Toxicol Pharmacol 33: 181-190, 2012.

15. Yan F, Wang M, Chen H, Su J, Wang X, Wang F, Xia L and Li Q: Gambogenic acid mediated apoptosis through the mitochondrial oxidative stress and inactivation of Akt signaling pathway in human nasopharyngeal carcinoma CNE-1 cells. Eur J Pharmacol 652: 23-32, 2011.

16. Yu XJ, Han QB, Wen ZS, Ma L, Gao J and Zhou GB: Gambogenic acid induces G1 arrest via GSK3 $\beta$-dependent cyclin D1 degradation and triggers autophagy in lung cancer cells. Cancer Lett 322: 185-194, 2012.

17. Peng C, Sun M, Su J and Li Q: Effect of gambogenic acid on inducing A549 cell apoptosis through regulating Ras/Raf/Erk signaling pathway. Traditional Chin Drug Res Clin Plarmacol 27: 189-193, 2016.

18. Su J, Cheng H, Zhang D, Wang M, Xie C, Hu Y, Chang HC and Li Q: Synergistic effects of 5-fluorouracil and gambogenic acid on A549 cells: Activation of cell death caused by apoptotic and necroptotic mechanisms via the ROS-mitochondria pathway. Biol Pharm Bull 37: 1259-1268, 2014.

19. Liu W, Guo QL, You QD, Zhao L, Gu HY and Yuan ST: Anticancer effect and apoptosis induction of gambogic acid in human gastric cancer line BGC-823. World J Gastroenterol 11: 3655-3659, 2005

20. Yi T, Yi Z, Cho SG, Luo J, Pandey MK, Aggarwal BB and Liu M: Gambogic acid inhibits angiogenesis and prostate tumor growth by suppressing vascular endothelial growth factor receptor 2 signaling. Cancer Res 68: 1843-1850, 2008.

21. Wang T, Wei J, Qian X, Ding Y, Yu L and Liu B: Gambogic acid, a potent inhibitor of survivin, reverses docetaxel resistance in gastric cancer cells. Cancer Lett 262: 214-222, 2008.

22. Wang K, Tang Y, Sun M, Lu B, Zhu H, Ji O and Shen Q: The mechanism of neogambogic acid-induced apoptosis in human MCF-7 cells. Acta Biochim Biophys Sin 43: 698-702, 2011.

23. Chen R, Zhang H, Liu P, Wu X and Chen B: Gambogenic acid synergistically potentiates bortezomib-induced apoptosis in multiple myeloma. J Cancer 8: 839-851, 2017.

24. Elmore S: Apoptosis: A review of programmed cell death. Toxicol Pathol 35: 495-516, 2007.

25. Jing G, Wang JJ and Zhang SX: ER stress and apoptosis: A new mechanism for retinal cell death. Exp Diabetes Res 2012: $589589,2012$.

26. Liu N, Huang H, Liu S, Li X, Yang C, Dou QP and Liu J: Calcium channel blocker verapamil accelerates gambogic acid-induced cytotoxicity via enhancing proteasome inhibition and ROS generation. Toxicol In Vitro 28: 419-425, 2014.

27. Li F, Wang Y and Yan Y: Gambogenic acid induces cell growth inhibition, cell cycle arrest and metastasis inhibition in choroidal melanoma in a dose-dependent manner. Exp Ther Med 13: 2456-2462, 2017.

This work is licensed under a Creative Commons Attribution-NonCommercial-NoDerivatives 4.0 International (CC BY-NC-ND 4.0) License. 